\title{
Gas breakdown by a focused beam of THz waves
}

\author{
A.V. Sidorov, S.V. Razin, A.G. Luchinin, A.I. Tsvetkov, A.P. Fokin, D.S. Sidorov, \\ A.P. Veselov, A.V. Vodopyanov, M.Yu. Glyavin
}

Institute of Applied Physics of Russian academy of Sciences, Nizhny Novgorod, Russia, sevraz@appl.sci-nnov.ru

\section{Introduction}

The sources of submillimeter radiation have made significant progress recently [1-3] and so the possibility to study gas discharge phenomena in terahertz wave beams has appeared. Such gas discharge is of great interest from viewpoint of both general and applied physics.

This paper presents the results of experimental and theoretical investigations of gas breakdown thresholds for several gases (argon, krypton, xenon, nitrogen, oxygen) in a focused beam of pulsed and $\mathrm{CW} \mathrm{THz} \mathrm{gyrotrons.} \mathrm{The} \mathrm{results} \mathrm{of} \mathrm{experiments} \mathrm{for}$ noble gases are in good agreement with theoretical calculations that were made based on a theory of breakdown of monatomic non-light gases in fields of any frequency from radio frequency to optical [4].

\section{Experimental Setups}

Two facilities with pulsed-mode [1] $(0.67 \mathrm{THz}$, $20 \mu \mathrm{s}, 40 \mathrm{~kW}$, Fig. 1 [5]) and CW-mode [3] (0.263 $\mathrm{THz}$, up to $1 \mathrm{~kW}$, Fig. 2) gyrotrons were used in the experiments. Terahertz radiation was focused into a vacuum chamber where discharge occurred by means of quasioptical mirrors. The intensity of the radiation in the focal spot was $16 \mathrm{MW} / \mathrm{cm}^{2}$ (rms electric field strength $80 \mathrm{kV} / \mathrm{cm}$ ) for a pulsed-mode gyrotron and up to $15 \mathrm{~kW} / \mathrm{cm}^{2}$ ( $\mathrm{rms}$ electric field strength 2.5 $\mathrm{kV} / \mathrm{cm}$ ) for a CW-mode gyrotron. Such electric field values were sufficient for the realization of a breakdown in the pressure range 1-1500 Torr.

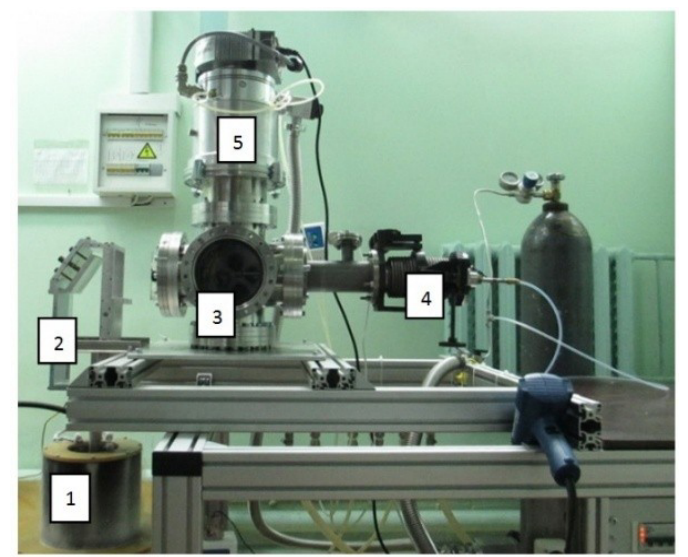

Fig. 1. Pulsed mode experimental setup (photo): 1 - pulsed gyrotron, 2 - quasioptical converter with focusing mirror, 3 discharge chamber with optical window, 4 - gas injection system, 5 - vacuum pumping system $(700 \mathrm{l} / \mathrm{s})$.

Several photos of the discharge obtained in different working gases and at different pressures at the facilities described above are shown in Figs. 3 and 4.

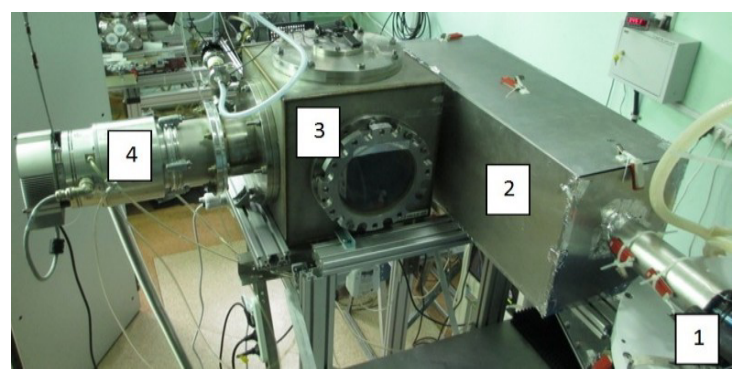

Fig. 2. CW mode experimental setup (photo): 1 - gyrotron output window, 2 - the protective cover of quasioptical beam line, 3 - discharge chamber, 4 - vacuum pumping system $(2000 \mathrm{l} / \mathrm{s})$.

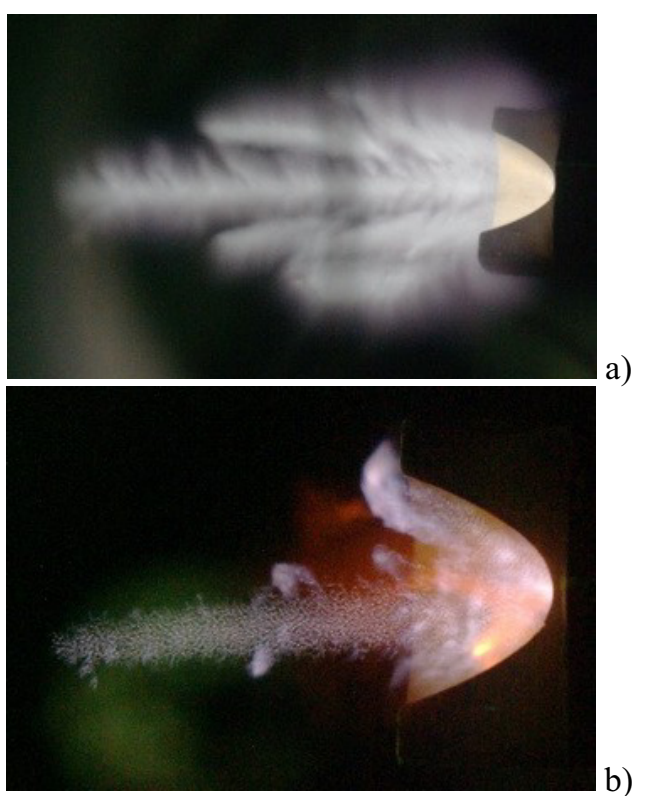

Fig. 3. Time-integrated photo of the emission of the pulsed discharge plasma in the visible spectral range: a) xenon, the pressure is 63 Torr; b) oxygen, the pressure is 760 Torr. The terahertz radiation is propagated from left to right.

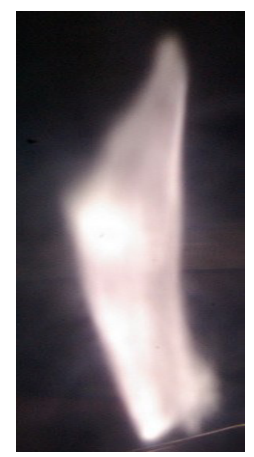

Fig. 4. Photo of the plasma glow of $\mathrm{CW}$ mode discharge in the visible spectral range. Argon, the pressure is 240 Torr, $\mathrm{THz}$ power is $950 \mathrm{~W}$. The terahertz radiation propagates from top to bottom. 


\section{Experimental Results}

The pressure breakdown thresholds for several gases in a focused beam of terahertz waves for a $\mathrm{THz}$ radiation power of $40 \mathrm{~kW}$ were determined during the experiments on a pulsed mode setup. The table shows the minimum gas pressures at which the discharge was observed.

\begin{tabular}{|c|c|c|c|c|}
\hline $\mathrm{Ar}$ & $\mathrm{Kr}$ & $\mathrm{Xe}$ & $\mathrm{N}_{2}$ & $\mathrm{O}_{2}$ \\
\hline 8 Torr & 1.5 Torr & 1.5 Torr & 12 Torr & 18 Torr \\
\hline
\end{tabular}

At pressures lower than those listed in the table, the discharge could not be ignited.

Fig. 5 shows the experimental dependence of the threshold $\mathrm{THz}$ radiation power at which the discharge occurred, on the argon pressure for the case of the CW mode setup. According to experiments, the minimum pressure at which it was possible to ignite the discharge was about 23 Torr. The minimum of the breakdown curve is reached at pressures of 50-60 Torr.

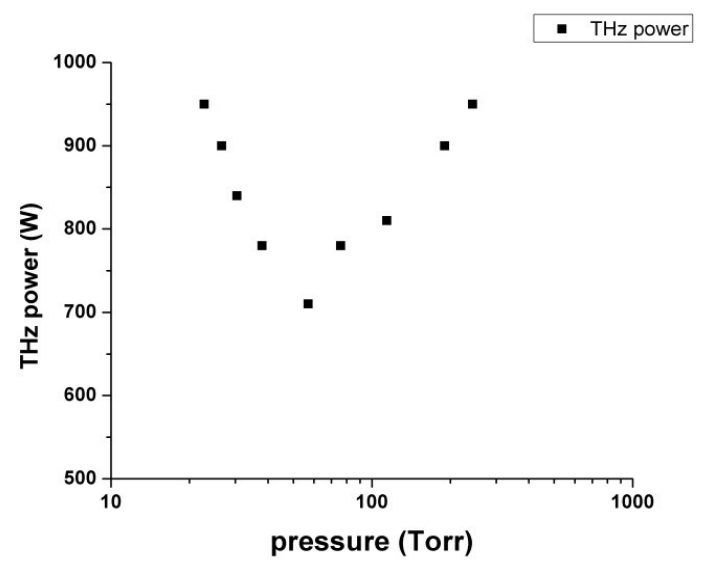

Fig. 5. Experimental threshold breakdown curve for Argon for $0.263 \mathrm{THz}$.

\section{Discussion and Conclusion}

Fig. 6 shows the calculated breakdown curve in comparison with the experimental one. The theoretical breakdown curve was calculated based on a theory of breakdown of monatomic non-light gases in electromagnetic field with frequency ranged from RF to optical [4]. The loss of electrons from the electric field area assumed to be diffusive with diffusion length $\Lambda$ of $0.06 \mathrm{~cm}$. The last one in our case was assumed as [6] $\Lambda=R / 2.4$, where $R=1.5$ $\mathrm{mm}$ - the radius of the wave beam in the focal spot. In this case, electron diffusion was assumed to be free.

The lower calculated curve corresponds to the diffusion length $\Lambda=0.6 \mathrm{~cm}$. Such a value can correspond to the conditions when electron diffusion is ambipolar [6]. In this case one can see a good agreement between calculations and experimental results (black spots).

Thus, experiments were performed to determine the breakdown thresholds of various gases in focused

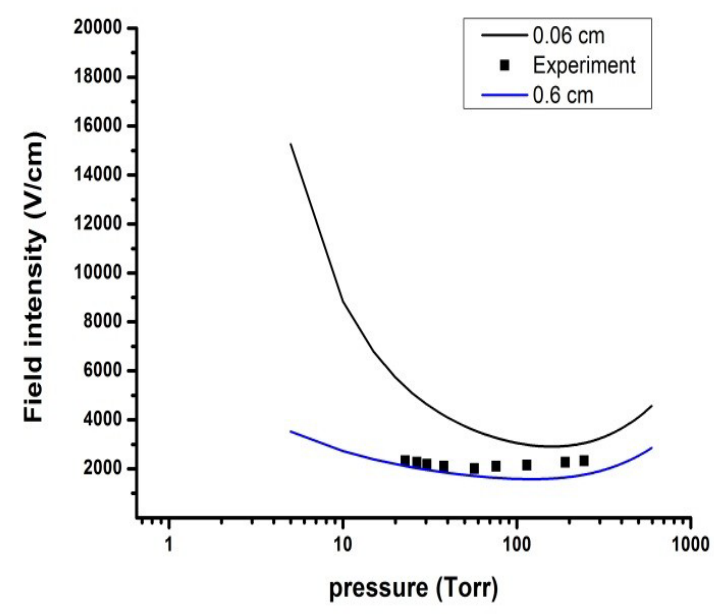

Fig. 6. Breakdown curves for $0.263 \mathrm{THz}$. Upper curve results of calculations for $\Lambda=0.06 \mathrm{~cm}$, lower curve $-\Lambda=0.6$ $\mathrm{cm}$. Black spots - experimental one.

beams of electromagnetic radiation with frequencies of $0.67 \mathrm{THz}$ and $0.263 \mathrm{THz}$ for the first time. Assuming that the electron losses are determined by diffusion, breakdown curves were calculated for the radiation frequency of $0.263 \mathrm{THz}$ in argon. The calculations are in good agreement with experiment for the case of ambipolar diffusion.

\section{Acknowledgements}

The work was supported by the Russian Science Foundation, project No. 14-12-00609.

\section{References}

1. M.Yu. Glyavin, A.G. Luchinin, G.S. Nusinovich, J. Rodgers, D.G. Kashyn, C.A. Romero-Talamas, and R. Pu. A $670 \mathrm{GHz}$ gyrotron with record power and efficiency. // Appl. Phys. Lett. 2012. V. 101. P. 153503.

2. V.L.Bratman, A.A.Bogdashov, G.G.Denisov, M.Yu.Glyavin, Yu.K.Kalynov, A.G.Luchinin, V.N.Manuilov, V.E.Zapevalov, N.A.Zavolsky, V.G.Zorin. Gyrotron development for high power THz technologies in IAP RAS. // Journal of Infrared, Millimeter, and Terahertz Waves. 2012. V. 33. No.7. P. 715.

3. M.Yu. Glyavin, A.V. Chirkov, G.G. Denisov, A.P. Fokin, V.V. Kholoptsev, A.N. Kuftin, A.G. Luchinin, G.Yu. Golubyatnikov, V.I. Malygin, M.V. Morozkin, V.N. Manuilov, M.D. Proyavin, A.S. Sedov, E.V. Sokolov, E.M. Tai, A.I. Tsvetkov, V.E. Zapevalov. Experimental tests of a $263 \mathrm{GHz}$ gyrotron for spectroscopic applications and diagnostics of various media. // Rev. Sci. Instrum. 2015. V. 86. No. 5. P. 54705.

4. A.I. Vyskrebentsev, Yu.P. Raizer. A simple theory of breakdown of monatomic nonlight gases in fields of any frequency from low to optical. // Journal of Appl. Mechanics and Techn. Phys. 1973. V. 14. P. 32.

5. M.Yu. Glyavin, S.V. Golubev, V.G. Zorin, I.V. Izotov, A.G. Litvak, A.G. Luchinin, M.V. Morozkin, S.V. Razin, A.V. Sidorov, V.A. Skalyga. The discharge maintained by highpower terahertz radiation in a nonuniform gas flow. // Radiophysics and Quantum Electronics. 2014. V. 56. No. 8-9. P. 561.

6. Yu.P. Raizer. Gas Discharge Physics. // Springer, New York 1991. 